\title{
Role of Insulin Signaling in the Pathophysiology of Alzheimer's Disease
}

\author{
Mini Dahiya, Anil Kumar* and Monu Yadav \\ University Institute of Pharmaceutical Sciences, Panjab University, India
}

Submission: October 17, 2020; Published: December 02, 2020

*Corresponding author: Dr Anil Kumar, Professor, Former-Dean of Faculty of Pharmaceutical Science, University Institute of Pharmaceutical Sciences, UGC Centre of Advanced Studies (UGC-CAS), Panjab University, Chandigarh, India

\begin{abstract}
Alzheimer's disease (AD) is a progressive neurodegenerative disorder demonstrated by brain deposition of $\mathrm{A} \beta$, which is responsible for the formation of senile plaques, neurofibrillary tangles (NFT) lead to neurodegeneration and cognitive alteration. Recently, it would be highlighted that alteration in insulin signaling pathways and glucose metabolism, in central along with peripheral systems, could be one of the prime reasons for the cognitive loss. Insulin resistance in the brain, also recognized as Type 3 diabetes, which further increases $\mathrm{A} \beta$ production and TAU phosphorylation, protein misfolding, mitochondrial dysfunction, oxidative stress, and cognitive impairment, which are all hallmarks of AD. Evidences indicated that $A \beta 42$ accumulation in the brain would induce hypothalamic dysfunction that leads to peripheral hyperglycemia due to downstream regulation of the insulin receptor in the liver. This review aim is to confer the existing facts which favor the concept that brain insulin resistance and altered glucose metabolism play an important role in the pathology of AD. Furthermore, we discuss AD treatment approaches along with the novel strategy of treating Alzheimer's and with drugs that initially have been developed to treat type 2 diabetes and mTOR inhibitors. As insulin signaling has been interrupted in the brains of AD patient, drugs that can resensitize insulin signaling have been studied to evaluate if this approach can alter disease prognosis.
\end{abstract}

Keywords: Alzheimer's disease; Diabetes mellitus; Insulin signaling, amyloid- $\beta$.

\section{Introduction}

Alzheimer's disease (AD) is progressive neurodegenerative disorder demonstrated by brain deposition of the amyloid $\beta$ peptide, insulin deficiency, neuroinflammation, oxidative damage, cholinergic loss lead to form neurofibrillary tangles (NFT) senile plaques and leads to neurodegeneration and cognitive impairment [1-3]. There are still several other factors like brain injury, diabetes, neurotoxicity and obesity, primarily responsible for the progression of AD. Though it has been a decade or more since the idea of diabetes mellitus (DM) as a causal marker for the development of various other neuronal disorder as well AD also, till now this relationship has not been well studied. As a chronic metabolic disorder, DM is the majority characterized by elevated blood glucose level due to insulin deficiency or loss of the insulin receptor sensitivity [4]. Since a decade, clinicians have identified the high morbidity and mortality rates associated with $\mathrm{AD}$ as compared to dementia. As per the data of the International Diabetes Federation, 425 million populations are suffering from diabetes worldwide and this amount probably increases to 629 million by the end of 2045 . Recent epidemiological data have indicated that patients with DM are more vulnerable to develop AD than a healthy person [5]. According to the World Health Organization (WHO) data by 2030 third world countries like India will contribute five times more as compared to developed countries to the prevalence of DM. This could be disquieting health crisis in developing countries as India, where inappropriate modern diagnostic tools and lack of suitable AD treatment approaches would contribute to this health crisis as well as other neuronal disorder including AD [6].

Cognitive dysfunction is the most distinctive symptom of several neurodegenerative disorders like Alzheimer's disease (AD), Multiple sclerosis, Huntington disease (HD) and Parkinson's disease (advanced stage). AD has been categories into two groups, based on onset, the first type is familial AD linked with genetic mutation of the amyloid-beta precursor protein (Amyloid Beta PP) and presenilins (PS1) [7]. Another group is the late-onset form (LOAD), also recognized as sporadic which is majority prevalent accounts for $97 \%$ of total diagnose [8]. Pathologically, AD includes selective pyramidal neuronal death and an accumulation of 
intraneuronal and extracellular fibrils, neurofibrillary tangles and senile plaques. Cognitive dysfunction develops due to abnormal accumulations of amyloid beta which is formed from the catabolism of amyloid-beta PP (plasmatic membrane protein of neurons and astrocytes), neuroinflammation, oxidative stress, protein misfolding, apolipoprotein E (APOE) gene and hyperphosphorylation of TAU protein in hippocampus and cerebral cortex region of the brain $[9,10]$. Recently, it has been found that any alteration in glucose metabolism and insulin signaling in central and peripheral systems would be one of the causes for the cognitive dysfunction. Insulin resistance in the brain is also referred to as Type 3 diabetes that would increase of amyloid-beta formation and TAU phosphorylation which become hallmarks for LOAD [11]. This article primarily focuses on the association between brain insulin resistance and cognition dysfunction involved in AD.

\section{Insulin Signaling in CNS (Central Nervous System)}

Insulin is a peptide hormone of 5.8-KDa which produced and released by the pancreatic $\beta$ cells in the blood vessel. Moreover, it is transported to peripheral as well as the brain by crossing the blood-brain barrier using a receptor-mediated mechanism. Moreover, the peripheral role of insulin is well explored, but there are few data about the insulin role in brain. Recently, various researches have shown the decrease of insulin mRNA inpatient with $\mathrm{AD}$ whereas de novo insulin synthesis in the brain is controversial till now. Therefore, it is hypothesized that maximum of brain insulin delivered by the peripheral tissues and the function of insulin in the brain is still uncertain. Although, researches demonstrated the widespread presence of insulin receptors (IR) in the brain especially in the hippocampus, cortex, hypothalamus and olfactory bulb showed an involvement of "neuroregulatory" mechanism for brain insulin signaling. Brain IR is enriched in neurons as compared to glia and in high quantity at the synapse. But, as peripherally, main role of brain IRs is not only glucose metabolism but also perform multiple functions such as alteration of synaptic plasticity, homeostatic regulation, neurotransmission and age-related neurodegeneration via various receptor mediate function such as apoptosis, calcium influx, neurotransmitter synthesis and synaptic connections and neurogenesis. It also regulates expression of GABA (gamma-aminobutyric acid), NMDA (N-methyl-D-aspartate) and AMPA ( $\alpha$-Amino-3- hydroxy5-methyl-4-isoxazolepropionic acid) mediated mechanisms which is responsible for LTP (long-term potentiation) and LTD (long-term depression). Physiologically, brain insulin bind to its receptor and stimulate a cascade mechanism to control important downstream kinases such as AKT/PKB, mTOR (mechanistic target of rapamycin), ERK (extracellular signal-related kinases), PI3K (phosphatidylinositol-3-kinase), glycogen synthase kinase $3 \beta$ (GSK3 $\beta$ ), BAD (BCL-2 agonist of cell death), FOX (fork-head box), MAPK (mitogen-activated protein kinase) are essential for neurons survival $[4,12,13]$. Moreover, insulin is critically entailed in the preservation and expansion of excitatory synapses and dendritic spine configuration via activation of PI3K/Akt/mTOR and Ras-related pathways which are integral to insulin signaling. Insulin also influences cell survival by altering apoptotic pathways. Therefore, interruption in insulin signaling of any of these pathways impair the neuronal performance and integrity which may result in the learning, memory and other defects lead to the development of AD.

\section{Insulin Resistance in the Brain: Alzheimer's Disease}

Brain insulin resistance referred to the state that insulin does not react efficiently to its physiological levels and leads to increase of $A \beta$ production and TAU phosphorylation, mitochondrial dysfunction, oxidative stress, protein misfolding, and cognitive dysfunction, which are all hallmarks of AD. Furthermore, key cellular mechanism involved in AD include insulin, IGF-1 (insulinlike growth factor 1), IRS1/2 (insulin receptor substrates 1 and 2) and crucial secondary messenger kinases like Akt and mTOR get inactivated, similarly as in diabetes peripherally. Insulin resistance in $\mathrm{AD}$ and diabetes resulting to increase the level of insulin thus, saturating insulin-degrading enzyme (IDE) for insulin and $A \beta$ degradation because both are amyloidogenic peptides have a same series of recognition motif. Recently, various studies indicated that the prevalence of $\mathrm{AD}$ is higher in T2D patients and obese individuals. IR is widely dispersed throughout the brain majorly in hippocampus, a neurogenic area involved in memory and learning. Insulin signaling plays important roles in synaptic development, neuronal growth, cell metabolism, mitogenesis, glucose utilization and all genes required for cellular proliferation [14]. Although insulin desensitization has also been observed in $\mathrm{AD}$ patient even, they did not have DM, it may be caused via chronic inflammation response and pro-inflammatory cytokines like tumor necrosis factor (TNF) will block growth factor signaling of insulin or IGF-1. Therefore, insulin signaling impairment leads to abnormal neuronal functioning supports the study that insulin resistance in the brain may promote LOAD. De la Monte et al., demonstrated that significant suppression of gene expression and insulin protein levels, IGF1 receptors and other downstream chemicals in the area of the hippocampus, frontal cortex, and hypothalamus along with reduced acetylcholine synthesis and cognitive function in a patient with $\mathrm{AD}$ [15].

\section{Relationship between IR signaling and Tau phosphor- ylation}

Physiologically, Tau protein acts as a microtubule-binding protein and involved in essential function in vesicular and axonal transport. While tau protein gets hyperphosphorylated and detached from the microtubules in the disease condition. Preclinical studies show that phosphor-tau mediated destruction of cell skeletal integrity involved in synaptic and behavioral alteration. Some in-vivo studies have established that tau phosphorylation can be induced by protein kinases and phosphatases like cyclin- 
dependent kinase 5 (CDK5), p25 (CDK5 regulator), active glycogen synthase kinase $3 \beta$ (GSK3 $\beta$ ) and its regulator c-JUN N-terminal kinase (JNK) are entailed in neurofibrillary pathogenesis and found upregulated in AD. Recent studies have been suggested that an insulin signaling impairment have the potential to raise the phosphorylation of tau protein which is one of the primary events in the development of AD. Hyperphosphorylated tau monomers mainly form NFTs which are clusters of paired helical filaments (PHF) consist of hyperphosphorylated tau protein which present in cell bodies and dendrites of neurons [16]. Insulin receptor (IR), insulin receptor substrate (IRS1) which further activate various cascading pathways which include PIP2 conversion into PIP3, further stimulation of PKB/AKT and, resulting into the translocation of GLUT4 transport to the plasma membrane $[17,18]$. Moreover, AKT signaling is responsible for neuronal survival and TAU phosphorylation. Other essential targets like GSK3 $\beta$ which are responsible for Tau phosphorylation, also regulated by AKT. Therefore, brain Insulin resistance leads to an increase in the activation of GSK3 $\beta$ resulting in hyperphosphorylation of TAU protein and the consequent development of neurofibrillary tangles is one of the main biomarker of $\mathrm{AD}[19,20]$.

\section{Role of GLUT 4 in Cognition}

GLUT4 (Glucose transporter 4) is present in peripheral tissues (adipose tissue, heart, skeletal muscle as well as in brain region like cerebellum, hippocampus. Physiologically, GLUT4 transport glucose to the cytoplasm from the extracellular space for its metabolism and controls energy needs for various neuronal activates upon the stimulation of the insulin receptor signaling cascade via insulin. Cognitive function of brain can be improved by increasing the GLUT4 induced glucose uptake. Therefore, upregulation of expression of GLUT4 in the hippocampus can be a potential target to treat the cognitive function in $\mathrm{AD}[21,22]$.

\section{Amyloid- $\beta$ Cascade Hypothesis}

Amyloid beta, a 40-42 amino acid proteolytic fragment which is generated from the catabolism of amyloid-beta PP, is a plasma membrane protein found in different cells such as astrocytes, neurons and oligodendrocytes and become a major constituent for amyloid plaques. In AD, irregular cleavage of APP leads to form insoluble amyloid- $\beta$ protein, compactly packed with betasheets, which is core of the senile plaques. The genetic studies that demonstrated that mutation in APP, PS1 and PS2 genes are responsible for deposition of amyloid-beta and leads to "Amyloid Cascade Hypothesis" [10,23]. Moreover, amyloid-beta tangles stimulate microglial leads to release of proinflammatory cytokines especially in the cortex and hippocampus [24]. Moreover, these tangles readily form dimmers and polymer and became a major toxic element for neuron death and their breakage. Amyloid-beta is produced by sequential cleavage of Amyloid Beta PP protein by $\alpha, \beta$, and $\gamma$ secretase enzymes along with a complex of multiprotein like PS1 and PS2 (Presenilin 1 and 2). Neuropathologically, amyloid-beta PP is metabolized via amyloidogenic pathway in which amyloid-beta PP cleaved by BACE $1(\beta$-secretase A $\beta$ cleaving enzyme 1$)$ by the $N$-terminal whereas $\gamma$-secretases cleave the $\mathrm{C}$-terminal leads to the formation of amyloid-beta 40 and amyloid-beta 42 fragments that release in the extracellular space and binds to apolipoprotein $\mathrm{E}$ (ApoE) $[9,25]$. These amyloid beta-ApoE binding will allow amyloid beta to undergo clearance mechanism by BBB, degradation by amyloid beta-degrading enzymes. Some hypothesis also indicated that diabetes is responsible for increasing the production of amyloidbeta and TAU phosphorylation, Type $2 \mathrm{DM}$ and amyloid-beta may result in neuron destruction in LOAD [4].

\section{Relationship of Amyloid Beta Peptide and Brain Insu-} lin

Insulin and amyloid-beta both are amyloidogenic peptides have the same series of recognition motif, so that there is a possibility both molecules may compete for binding to the insulin receptor. This hypothesis suggests that amyloid-beta have potential to resist insulin in brain via their antagonistic property, resulting in the inhibition of the downstream pathway and activating the GSK3 $\beta$ phosphorylation which further responsible for amyloid-beta production and hyperphosphorylation of TAU lead to neurodegeneration [26]. The intracerebral injected amyloid-beta develop glucose intolerance peripherally and brain insulin resistance and affect other mechanisms such as release of inflammatory parameter in hypothalamus and adipose tissue, altered GLUT-4 translocation to cell membrane induced by insulin in skeletal muscle. A recent report demonstrated that there can be known as Factor $\mathrm{X}$ which may be a molecule or pathway that will form a bridge to connect amyloid beta accumulation as a responsible factor for T2DM and LOAD. Additionally, the authors suggest that Factor $\mathrm{X}$ characterization will be an important parameter to develop an efficient therapeutic target for LOAD treatment [27]. The connection between insulin resistance, amyloid Beta and tau phosphorylation in the progression of $\mathrm{AD}$ has been shown in Figure 1.

\section{BACE1 an Important Connection Between T2DM and}

\section{AD}

$\beta$-secretase amyloid-beta cleaving enzyme 1 (BACE1) is an enzyme and a rate-limiting element in amyloid-beta formation. It has been reported that amyloid-beta (1-42) increase the BACE1 gene expression via MAPK8/JNK1-MAPK9/JNK2 signaling pathway. Recent preclinical studies showed that neuronal overexpression of human BACE1 develop systemic diabetic by hypothalamic impairment, hepatic deficits, insulin resistance, and glucose alterations [28,29]. It has been reported that overexpression of BACE-1 in neurons increase the risk 
and providing a link to understand a complex mechanistic interaction between LOAD and T2DM. Though, neuronal BACE1 is responsible for metabolic dysfunction in all over the body including brain inflammatory response and cognitive alteration due to amyloidosis. The present hypothesis suggest that hyperglycemia initially can occur in the brain and adversely affect the remaining body. These finding highlights a new and potential target like BACE1 role in metabolic process and suggest a potential bridge between T2DM and AD [30] (Table 1).

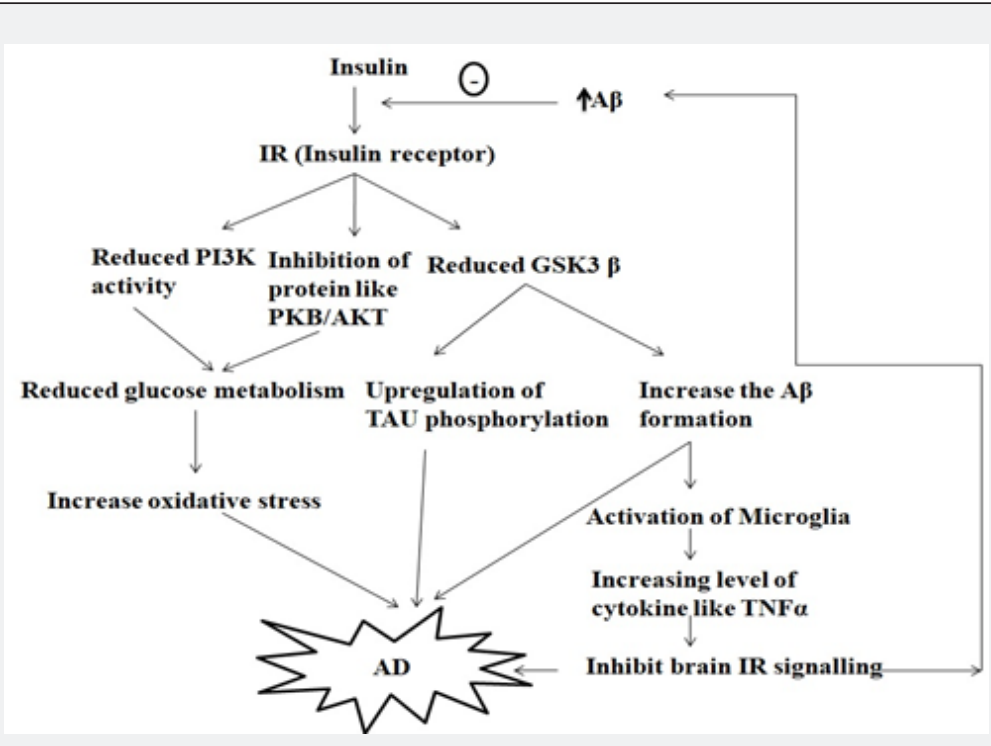

Figure 1: Outcome of insulin, amyloid-beta, TAU phosphorylation crosstalk on neuronal IR signaling.

Table1: IR signaling pathway alterations in the brain in late-onset Alzheimer's disease.

\begin{tabular}{|c|c|c|}
\hline Physiological impairment & Pathological effects & Reference \\
\hline $\begin{array}{l}\text { Insulin modulates levels of acetylcholine and norepinephrine neu- } \\
\text { rotransmitters }\end{array}$ & Memory loss & [31] \\
\hline Formation and deposition of advanced glycation end products (AGEs) & $\begin{array}{c}\text { Upstream regulation of APP via oxidative stress and } \mathrm{A} \beta \\
\text { accumulation }\end{array}$ & [32] \\
\hline $\begin{array}{l}\text { Decline in ATP levels (mitochondrial alteration). PKB activity inhibi- } \\
\text { tion GSK3 activity increase }\end{array}$ & $\begin{array}{l}\text { Amount in TAU phosphorylation. Oxidative stress } \\
\text { increases }\end{array}$ & [33] \\
\hline Insulin resistance inhibits IDE activity & $A \beta$ levels Increase & [34] \\
\hline resistance increases vascular dysfunction & Vascular dementia & [35] \\
\hline mTOR dysregulation & Learning and memory deficits, cell cycle reentry & [36] \\
\hline $\begin{array}{l}\text { Neuroinflammation and TNF } \alpha \text { increase associated with neuronal ER } \\
\text { stress and JNK activation }\end{array}$ & Brain IR down-regulation and synaptic alteration & [37] \\
\hline $\begin{array}{c}\text { Hippocampal-specific insulin resistance using a lentiviral vector } \\
\text { expressing an IR antisense sequence }\end{array}$ & $\begin{array}{l}\text { Downregulation of GluN2B and GluA1 phosphorylation } \\
\text { at synapses. Memory failure independent of peripheral } \\
\text { metabolic alterations }\end{array}$ & [38] \\
\hline $\begin{array}{l}\text { Downregulation in neurogenesis was associated with reductions in } \\
\text { dendritic spine density in CA1 pyramidal neurons }\end{array}$ & Learning and memory loss & [39] \\
\hline
\end{tabular}

\section{AD potential therapeutically approaches related to the insulin metabolism regulation}

AD treatment which includes FDA-approved choline esterase inhibitors and NMDA (glutamate) receptor agonists is still at a nascent stage shows only symptomatic relief, but they are not decreasing the progression of the AD.

\section{Intranasal insulin for LOAD treatment}

Preclinical, intranasal administration of insulin /insulin analogues has the potential to improve memory in mice models of AD. Moreover, Craft and co-workers research studies show that administration of intranasal-insulin improves memory for adults with mild cognitive impairment and LOAD. Additionally, 
the authors suggest that insulin could improve and modify the AD-related pathophysiologic processes. Therefore, the above data support the continued investigation of the potential stimulation of the insulin receptor as a therapeutic approach for LOAD [40-42].

\section{Pioglitazone: a modulator of PPAR $\gamma$ Receptor (Proliferation of Activated Gamma Peroxisome)}

Pioglitazone is an orally active anti-diabetic drug belongs to thiazolidinediones family, also referred to as "insulin sensitizers". It is a selective and potent receptor agonist for the PPAR $\gamma$ receptor $[43,44]$. These receptors control the transcription of several genes that are sensitive to insulin. PPAR $\gamma$ are located in various tissues such as adipose tissue, skeletal muscle and liver where insulin exerts its effect. Activation of PPAR $\gamma$ receptors mediate the transcription of the gene that is entailed in the regulation of glucose transport, production and its utilization. In LOAD, pioglitazone has been shown to decrease the pro-inflammatory activity of glial cell and, to reduce $A \beta$ peptide levels due to the microglial phagocytic activity. Treatment with pioglitazone in 3xTg-AD mice model for 4 months, and result showed that drug improves TAU hyperphosphorylation, cognitive impairment, and neuroinflammation [43]. Recently, Fernandez-Martos and coworkers study indicated that the combination of pioglitazone and leptin demonstrated the useful effects in the mice model having familial AD improving memory and suppressing $A \beta$ levels [45]. Therefore, it has been observed that pioglitazone have the potential to reverse the loss of synaptic density caused by $A \beta$ accumulation. Moreover, preclinical data support the beneficial effects of pioglitazone in $\mathrm{AD}$ whereas, clinical study till now indicated somehow conflicting results regarding efficacy due to various limitations of these trials. Clinical trials on the potential use of pioglitazone for the treatment of LOAD are necessary. Furthermore, clinical trials (Phase II) of the drug indicated that it is a safe and well-tolerated drug [46].

\section{Rapalogs}

It well explores pathway that is $\mathrm{PI} 3 \mathrm{~K} / \mathrm{AKT} / \mathrm{mTOR}$ and its dysregulation may reduce the autophagic process which is responsible for the aggregation of $A \beta 42$ and protein accumulation. Like, mTOR is entailed in the alteration of IRS1 activity, showing one of the well-characterized parts leading to insulin resistance [47]. Therefore, mTOR activation pathway could be an essential link between insulin signaling and $A \beta$ and contribute to new insights to insulin resistance relationship with the incidence of AD. Both rapamycin and its derivatives inhibit mTORC1 complex formation, acting as allosteric inhibitors. Whereas a major disadvantage of rapamycin are its long half-life, solubility, and the poor oral absorption making it necessary for the development of rapamycin analogue such as ester derived temsirolimus. Its use was approved in 1977 by the FDA and the European Medicines Agency for the treatment of renal carcinoma [48]. Both drugs have the same mechanism of action. Jiang and co-workers recently reported that temsirolimus promotes autophagic clearance of $A \beta$, exerts protective effects accompanied by an improvement in spatial cognitive functions in the APP/PS1 model of familial AD. This study gives support to the therapeutic potentials of temsirolimus in preclinical models of AD [45].

Novel Emerging Strategies to Treat AD: GLP-1 (incretin hormone glucagon-like peptide -1) and GIP (Glucose-Dependent Insulinotropic Polypeptide)

\section{GLP-1 analogues}

Agonists of the incretin hormone glucagon-like peptide 1 (GLP-1) receptor are effective and well-tolerated drug treatments for T2DM. GLP-1 is part of the peptide growth factor family and its downstream signaling effects on cell growth and repair mechanism as compared to insulin. Nigel Greig et al. were the first to test GLP-1 analogues in preclinical studies to explore that they have a neuroprotective effect. GLP-1 receptors are found in the brain, where they possess growth factor-like effects and inhibit programmed cell death of neuronal cells [49]. Preclinical studies showed that mice have an abundance of GLP-1 receptors excess in the hippocampus which is responsible for increasing neuronal growth rate, survival and improved learning abilities. The use of GLP1 agonists such as liraglutide, lixisenatide and semaglutide that is drugs used for T2DMhave neuroprotective effect and can cross BBB as desired property for the treatment of AD. Moreover, exendin-4 (GLP-1 receptor agonists) showed good neuroprotective effects in a triple transgenic mouse AD model that expresses APP, PS1, tau human mutated genes related to early-onset AD. Currently, exendin4 is used for the treatment of T2DM and showed neuroprotective effects in various animal models [50,51].

\section{Glucose-dependent insulinotropic polypeptide (GIP) analogues are protective in animal models of Alzheimer's disease}

GIP is the incretin mimetic hormone as GLP-1 and physiologically their roles are closely similar. It contains a chain of 42 amino acid expressed in various cells including neurons. The GIP receptor is a G-protein coupled receptor (GPCR) of the glucagon type family that increase cAMP levels like GLP-1, GIP receptor expression has been found in large neurons such as the pyramidal neurons in the cortex and hippocampus, granule neurons in the dentate gyrus, Purkinje cells in the cerebellum, and basal brain areas. Moreover, GIP analogues in the APP/PS1 mouse model of AD shows that improvement in synaptic plasticity in the hippocampus, DNA damage and Oxidative stress also decreased by the drug. Direct infusion of native GIP prevents the memory impairments caused intravenous injection of amyloid. Moreover, results suggest that GIP receptor activation has similar protective properties as GLP-1 receptor activation and that improving GIP signaling in the brain may be protective in $\mathrm{AD}$ [51]. 


\section{Conclusion}

This review is focused on the pathology of AD is very closely associated with the impairment in insulin signaling. The healthy functioning of cell depends on the effective energy metabolism, when it gets disturbed, affects adversely cellular function. Moreover, glucose is the prime energy source for the neurons that is why the IR has such a major role. Recently it has been well-explored mechanism that in hippocampus $A \beta$ could bind to the IR is become a major factor for memory loss, whenever the IR is blocked and elevate the neurodegenerative in this brain area. Therefore, antidiabetic treatment like intranasal insulin or pioglitazone more may be effective against AD. Additionally, new emerging strategies GLP-1 and GIP mimetic agent clinically used for T2DM have been shown neuroprotective effect in the animal model of AD. Furthermore, the therapeutic potential of BACE 1 inhibitors in reducing brain $A \beta$ plaque formation has not yet been confirmed clinically. Before widespread cognitive loss due to synapses and neurons degeneration, treatment approach of $\mathrm{AD}$ would produce the best clinical results, but recognition of these individuals at the early stage of $\mathrm{AD}$ is quite difficult. Accordingly, the modulation of brain IR inhibiting its inactivation could be a well-suited approach in a combinatory strategy therapy for AD.

\section{Acknowledgement}

The authors are thankful to for the financial support of SERB-DST (New Delhi) Letter number PDF/2018/002605 and University Institute of Pharmaceutical Sciences (UIPS) for the infrastructure facilities to carry out this work.

\section{References}

1. Razay G, Vreugdenhil A, Wilcock G (2006) Obesity, abdominal obesity and Alzheimer disease. Dement Geriatr Cogn Disord 22(2): 173-176.

2. Crane PK, Walker R, Hubbard RA, Li G, Nathan DM, Zheng H, et al. (2013) Glucose levels and risk of dementia. N Engl J Med 69(6): 540548.

3. Ikram MA, Brusselle GGO, Murad SD, van Duijn CM, Franco OH, et al. (2017) The rotterdam study: 2018 update on objectives, design and main results. Eur J Epidemiol 32(9): 807-850.

4. Folch J, Ettcheto M, Busquets O, Sanchez-Lopez E, Castro-Torres RD, Verdaguer $E$ et al. (2018) The implication of the brain insulin receptor in late onset Alzheimer's disease dementia. Pharmaceuticals 11(1): 11.

5. Sun Y, Ma C, Sun H, Wang H, Peng W, Zhou Z et al. (2020) Metabolism: A Novel Shared Link between Diabetes Mellitus and Alzheimer's Disease. J Diabetes Res.

6. Madhusudhanan J, Suresh G, Devanathan V (2020) Neurodegeneration in type 2 diabetes: Alzheimer's as a case study. Brain Behav 10(5): p.e01577.

7. Parihar MS, Hemnani T (2004) Alzheimer's disease pathogenesis and therapeutic interventions. J Clin Neurosci 11(5): 456-467.

8. Toodayan N (2016) Professor Alois Alzheimer (1864-1915): Lest we forget. J Clin Neurosci 31:47-55.

9. Selkoe DJ (2011) Resolving controversies on the path to Alzheimer's therapeutics. Nat Med 17(9):1060-1065.
10. Selkoe DJ, Hardy J (2016) The amyloid hypothesis of Alzheimer's disease at 25 years. EMBO Mol Med 8(6):595-608.

11. Steen E, Terry BM, Rivera EJ, Cannon JL, Neely TR, Tavares R et al. (2005) Impaired insulin and insulin-like growth factor expression and signaling mechanisms in Alzheimer's disease-Is this type3 diabetes? J Alzheimer's Dis 7(1): 63-80.

12. McCrimmon RJ, Ryan CM, Frier BM (2012) Diabetes and cognitive dysfunction. Lancet 379(9833): 2291-2299.

13. Cholerton B, Baker LD, Montine TJ, Craft S (2016) Type 2 diabetes, cognition, and dementia in older adults: toward a precision health approach. Diabetes Spectr 29(4): 210-219.

14. Hölscher C (2020) Brain insulin resistance: role in neurodegenerative disease and potential for targeting. Expert Opin Investig Drugs 29(4): 333-348.

15. De la Monte, SM (2014) Type 3 diabetes is sporadic Alzheimer's disease: Mini-review. Eur Neuropsychopharmacol 24(12): 1954-1960.

16. Palmer AM (2011) Neuroprotective therapeutics for Alzheimer's disease: progress and prospects. Trends Pharmacol Sci 32(3): 141147.

17. Ribe EM, Lovestone S (2016) Insulin signalling in Alzheimer's disease and diabetes: from epidemiology to molecular links. J Intern Med 280(5): 430-442.

18. Stanley M, Macauley S.L, Holtzman DM (2016) Changes in insulin and insulin signaling in Alzheimer's disease: Cause or consequence? J Exp Med 213(8):1375-1385.

19. Liu Y, Liu F, Grundke-Iqbal I, Iqbal K, Gong CX (2011) Deficient brain insulin signalling pathway in Alzheimer's disease and diabetes. J Pathol 225(1):54-62.

20. Pardeshi R, Bolshette N, Gadhave K, Ahire A, Ahmed S, Cassano T et al. (2017) Insulin signaling: An opportunistic target to minify the risk of Alzheimer's disease. Psychoneuroendocrinology 83:159-171.

21. Pearson-Leary J, McNay EC (2016) Novel Roles for the InsulinRegulated Glucose Transporter-4 in Hippocampally Dependent Memory. J Neurosci 36(47): 11851-11864.

22. Mehta V, ParasharA,Sharma A, Singh T.R, Udayabanu M (2017) Quercetin ameliorates chronic unpredicted stress-mediated memory dysfunction in male Swiss albino mice by attenuating insulin resistance and elevating hippocampal GLUT4 levels independent of insulin receptor expression. Horm Behav 89: 13-22

23. Christiane Reitz (2012) Alzheimer's disease and the amyloid cascade hypothesis: a critical review. Int J Alzheimer's Dis 1-11.

24. Prakash A, Kumar A (2014) Implicating the role of lycopene in restoration of mitochondrial enzymes and BDNF levels in $\beta$-amyloid induced Alzheimer's disease. Eur J Pharmacol 741: 104-111.

25. Vishal S, Sourabh A, Harkirat S (2011) Alois Alzheimer (1864-1915) and the Alzheimer syndrome. J Med Biogr 19(1): 32-33.

26. Kang S, Lee YH, Lee JE (2017) Metabolism-Centric Overview of the Pathogenesis of Alzheimer's disease. Yonsei Med J 58(3): 479-488.

27. Shinohara M, Sato N (2017) Bidirectional interactions between diabetes and Alzheimer's disease. NeurochemInt 108: 296-302.

28. Guglielmotto M, Monteleone D, Giliberto L, Fornaro M, Borghi R, Tamagno E et al. (2011) Amyloid- $\beta_{42}$ activates the expression of BACE1 through the JNK pathway. J Alzheimers Dis 27(4):871-883.

29. Roher AE, Esh CL, Kokjohn TA, Castaño EM, Van Vickle GD, et al. (2009) Amyloid beta peptides in human plasma and tissues and their significance for Alzheimer's disease. Alzheimers Dement. 5(1): 18-29. 
30. Meakin PJ, Harper AJ, Hamilton DL, Gallagher J, McNeilly AD, et al. (2012) Reduction in BACE1 decreases body weight protects against diet-induced obesity and enhances insulin sensitivity in mice. Biochem J 441(1): 285-296.

31. Hoyer S (1994) Neurodegeneration Alzheimer's disease and betaamyloid toxicity. Life Sci 55(25-26): 1977-1983.

32. Frolich L, Blum-Degen D, Riederer P, Hoyer S (1999) A disturbance of the neuronal insulin receptor signal transduction in sporadic Alzheimer's disease. Ann N Y Acad Sci 893:290-294.

33. Hoyer S (2004) Glucose metabolism and insulin receptor signal transduction in Alzheimer disease. Eur J Pharmacol 490(1-3): 115125.

34. Craft S (2005) Insulin resistance syndrome and Alzheimer's disease: Age- and obesity-related effects on memory, amyloid, and inflammation. Neurobiol Aging 26(1): 65-69.

35. Craft S (2012) Alzheimer disease: Insulin resistance and AD: Extending the translational path. Nat Rev Neurol 8(7): 360-362.

36. De Felice FG, Ferreira ST (2014) Inflammation, defective insulin signaling, and mitochondrial dysfunction as common molecular denominators connecting type 2 diabetes to Alzheimer Disease. Diabetes 63(7): 2262-2272.

37. De Felice FG, Lourenco MV, Ferreira ST (2014) How does brain insulin resistance develop in Alzheimer's disease? Alzheimer's Dement 10(1): S26-S32.

38. Grillo CA, Piroli GG, Lawrence RC, Wrighten SA, Green AJ, Wilson SP et al. (2015) Hippocampal Insulin Resistance Impairs Spatial Learning and Synaptic Plasticity. Diabetes 64(11): 3927-3936.

39. Biessels GJ, Reagan LP (2015) Hippocampal insulin resistance and cognitive dysfunction. Nat Rev Neurosci 16(11):660-671.

40. Craft S, Baker LD, Montine TJ, Minoshima S, Watson GS, Claxton A et al. (2012) Intranasal insulin therapy for Alzheimer disease and amnestic mild cognitive impairment: A pilot clinical trial. Arch Neurol 69(1): 29-38.

41. Bergmann L, Maute L, Guschmann M (2014) Temsirolimus for advanced renal cellcarcinoma. Expert Rev Anticancer Ther 14: 9-21.
42. Craft S, Claxton A, Baker LD, Hanson AJ, Cholerton B, Trittschuh EH et al. (2017) Effects of Regular and Long-Acting Insulin on Cognition and Alzheimer's Disease Biomarkers: A Pilot Clinical Trial. J Alzheimers Dis 57(4): 1325-1334.

43. Hölscher C (2014) Drugs developed for treatment of diabetes show protective effects in Alzheimer's and Parkinson's diseases. Sheng Li Xue Bao 66(5): 497-510.

44. Sato T, Hanyu H, Hirao K, Kanetaka H, Sakurai H, Iwamoto T (2011) Efficacy of PPAR-gamma agonist pioglitazone in mild Alzheimer disease. Neurobiol Aging 32(9): 1626-1633.

45. Fernandez-Martos CM, Atkinson RAK, Chuah MI, King AE, Vickers, JC (2016) Combination treatment with leptin and pioglitazone in a mouse model of Alzheimer's disease.Alzheimers Dement. 3(1): 92-106.

46. Galimberti D, Scarpini E (2017) Pioglitazone for the treatment of Alzheimer's disease. Expert Opin Investig Drugs 26(1): 97-101.

47. Di Domenico F, Barone E, Perluigi M, Butterfield DA (2017) The Triangle of Death in Alzheimer's Disease Brain: The Aberrant Cross-Talk Among Energy Metabolism Mammalian Target of RapamycinSignaling and Protein Homeostasis Revealed by Redox Proteomics. Antioxid Redox Signal 26:3 64-387.

48. Kwitkowski VE, Prowell TM, Ibrahim A, Farrell AT, Justice R, et al. (2010) FDA approval summary: Temsirolimus as treatment for advanced renal cell carcinoma. Oncologist 15(4): 428-435.

49. Tai J, Liu W, Li Y, Li L, Holscher C (2018) Neuroprotective effects of a triple GLP-1/GIP/glucagon receptor agonist in the APP/PS1 transgenic mouse model of Alzheimer's disease. Brain Res 1678: 64-74.

50. Bertilsson G, Patrone C, Zachrisson O et al. (2008) Peptide hormone exendin-4 stimulates subventricular zone neurogenesis in thce adult rodent brain and induces recovery in an animal model of Parkinson's disease. Neurosci Res 86(2): 326-38.

51. Holscher C (2018) Novel dual GLP-1/GIP receptor agonists show neuroprotective effects in Alzheimer's and Parkinson's disease models. Neuropharmacology 136(Part-B): 251-259.

52. Shi L, Zhang Z, Li L, Holscher C (2017) A novel dual GLP-1/GIP receptor agonist alleviates cognitive decline by re-sensitizing insulin signaling in the Alzheimer ICV: STZ rat model. Behav Brain Res 327: 65-74.

\section{Your next submission with Juniper Publishers will reach you the below assets}

- Quality Editorial service

- Swift Peer Review

- Reprints availability

- E-prints Service

- Manuscript Podcast for convenient understanding

- Global attainment for your research

- Manuscript accessibility in different formats

( Pdf, E-pub, Full Text, Audio)

- Unceasing customer service

Track the below URL for one-step submission

https://juniperpublishers.com/online-submission.php 